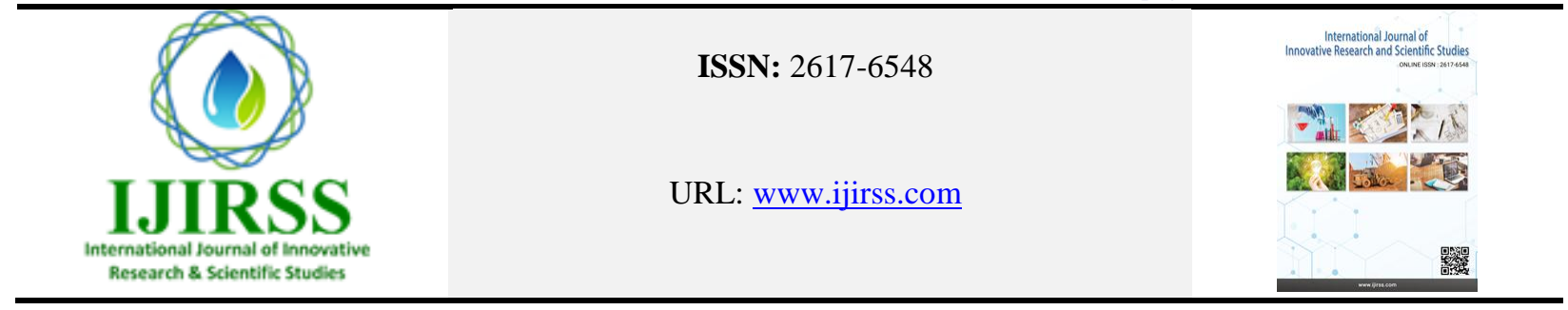

\title{
Heat Pump Systems: A Mini Review
}

\author{
Mohammad Omar Temori ${ }^{1 *}$, František Vranay ${ }^{2}$ \\ ${ }^{l}$ Faculty of Civil Engineering, Bakhtar University, Kabul, Afghanistan \\ ${ }^{2}$ Faculty of Civil Engineering, Technical University of Košice, Košice, Slovak Republic \\ *Corresponding author: Mohammad Omar Temori (dean.bce@bakhtar.edu.af)
}

\begin{abstract}
In this work, a mini review of heat pumps is presented. The work is intended to introduce a technology that can be used to income energy from the natural environment and thus reduce electricity consumption for heating and cooling. A heat pump is a mechanical device that transfers heat from one environmental compartment to another, typically against a temperature gradient (i.e. from cool to hot). In order to do this, an energy input is required: this may be mechanical, electrical or thermal energy. In most modern heat pumps, electrical energy powers a compressor, which drives a compression - expansion cycle of refrigerant fluid between two heat exchanges: a cold evaporator and a warm condenser. The efficiency or coefficient of performance (COP), of a heat pump is defined as the thermal output divided by the primary energy (electricity) input. The COP decreases as the temperature difference between the cool heat source and the warm heat sink increases. An efficient ground source heat pump (GSHP) may achieve a COP of around 4. Heat pumps are ideal for exploiting low-temperature environmental heat sources: the air, surface waters or the ground. They can deliver significant environmental $\left(\mathrm{CO}_{2}\right)$ and cost savings.
\end{abstract}

Keywords: Heat pump, evaporators, condensers, compressors, expansion wall, drive power, energy transfer, refrigerant, medium, coefficient of performance, seasonal performance factor, primary energy rate.

DOI: 10.53894 /ijirss.v4i2.59

Funding: This study received no specific financial support.

History: Received: 15 January 2021/Revised: 24 February 2021/Accepted: 22 March 2021/Published: 30 March 2021

Licensed: This work is licensed under a Creative Commons Attribution 4.0 License $($ (c) $)$ EY

Acknowledgement: Both authors contributed to the conception and design of the study.

Competing Interests: The authors declare that they have no conflict of interests.

Transparency: The authors confirm that the manuscript is an honest, accurate, and transparent account of the study was reported; that no vital features of the study have been omitted; and that any discrepancies from the study as planned have been explained.

Ethical: This study follows all ethical practices during writing. 


\title{
سيستم هاى بِمب حرارتى: يك بررسى كوتاه
}

\author{
محمد عمر تيمورى1، فر انتشيك وران اى2.

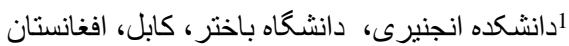

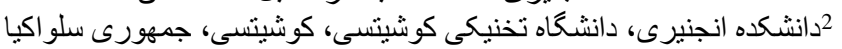

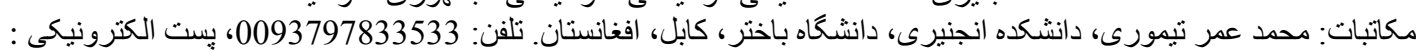 \\ dean.bce@bakhtar.edu.af
}

\begin{abstract}
جكيده

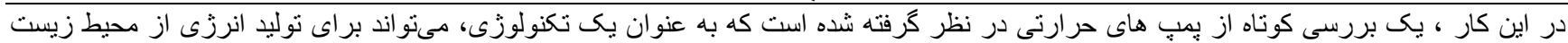

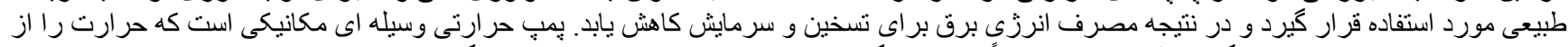

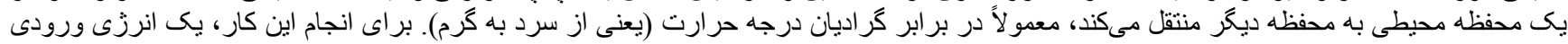

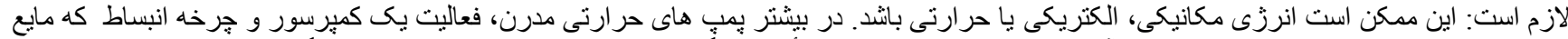

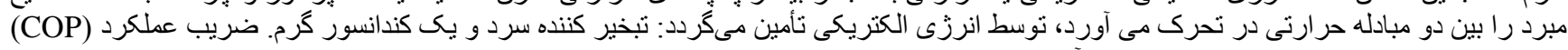

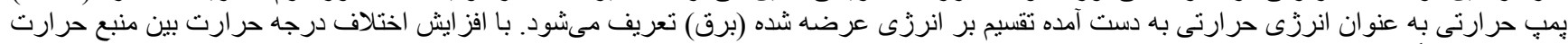

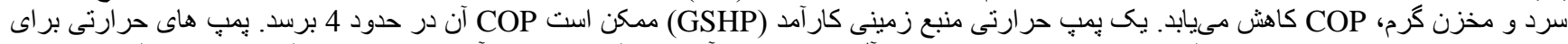

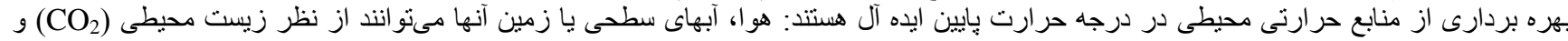

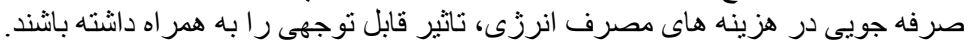

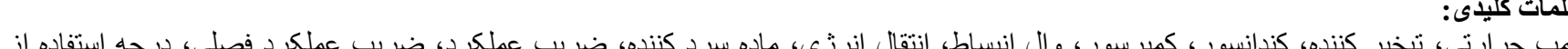

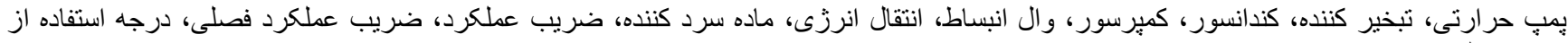
انرزّى اوليه.

1 1

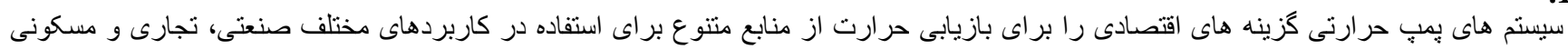

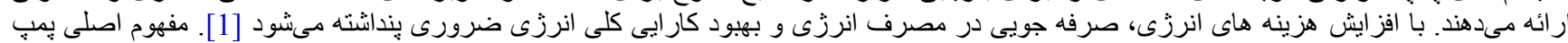

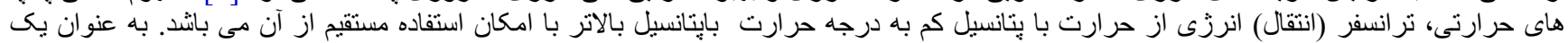

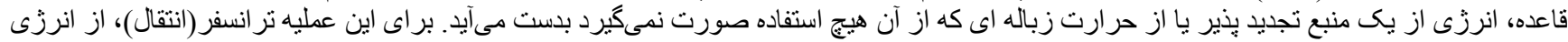

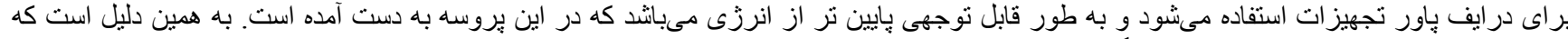

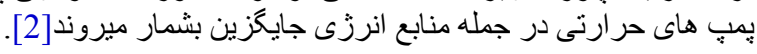

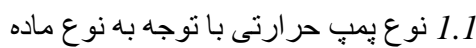

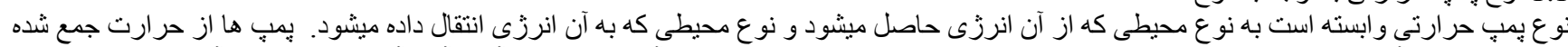

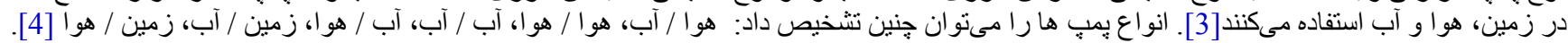

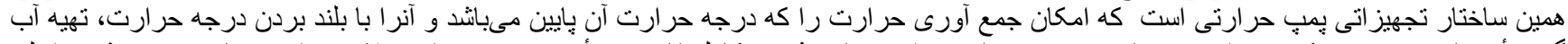

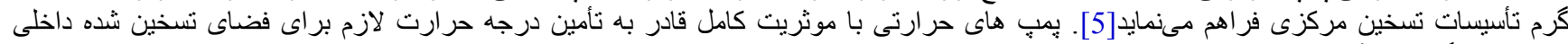

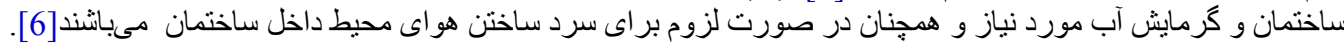

1.2

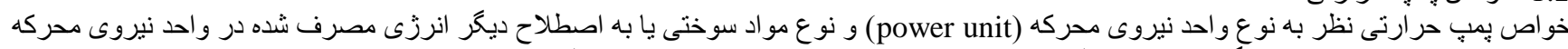

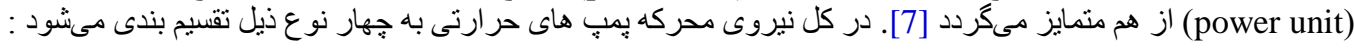

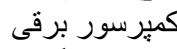

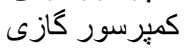

جاذب (Absorbent)

جذب سطحى (Adsorption)

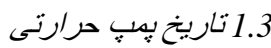

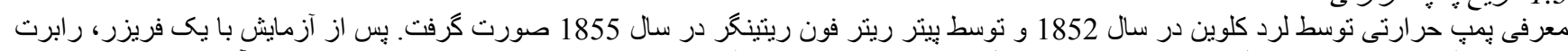

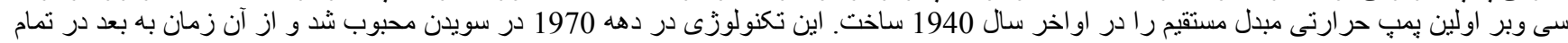

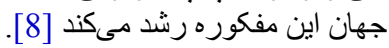

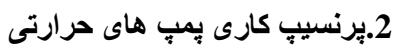

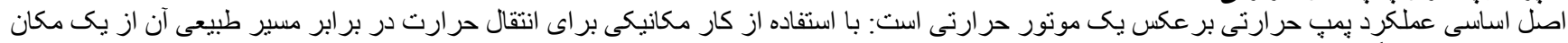

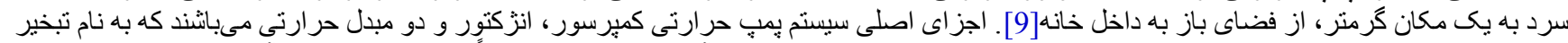

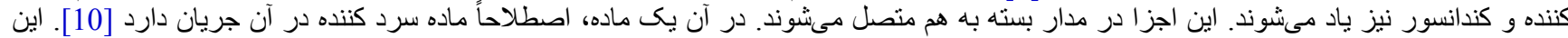

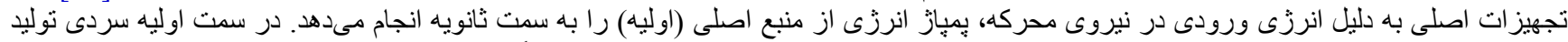

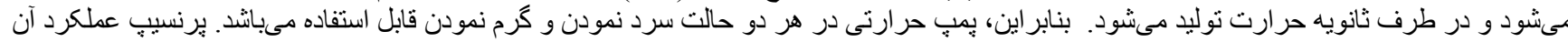

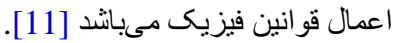


2.1

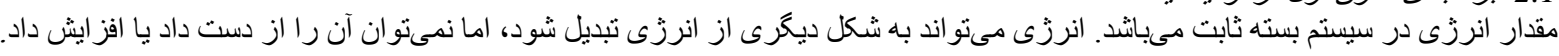

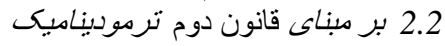

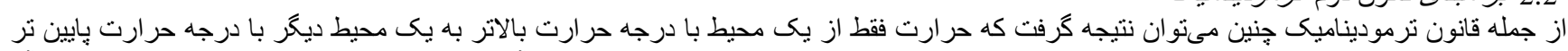

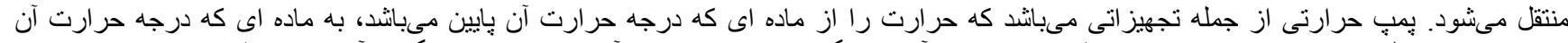

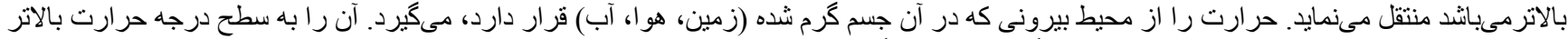

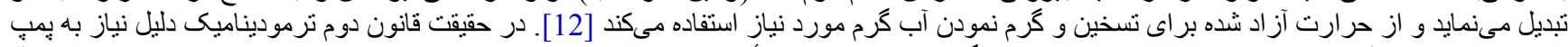

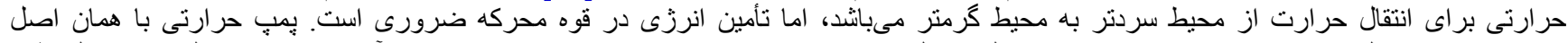

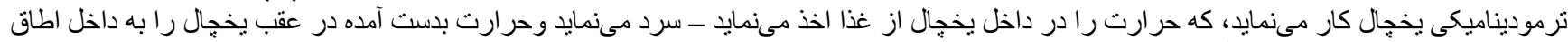

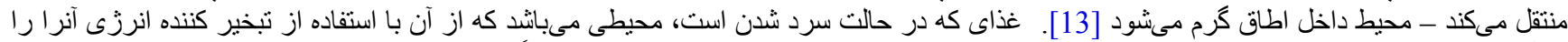

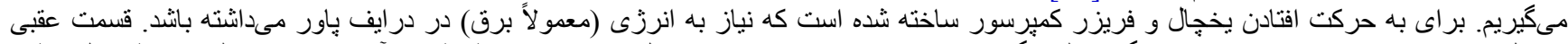

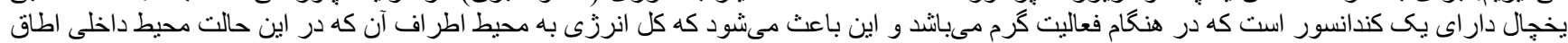

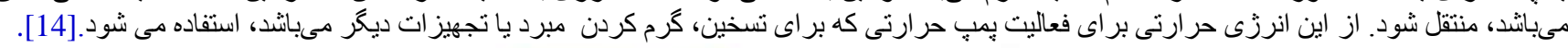

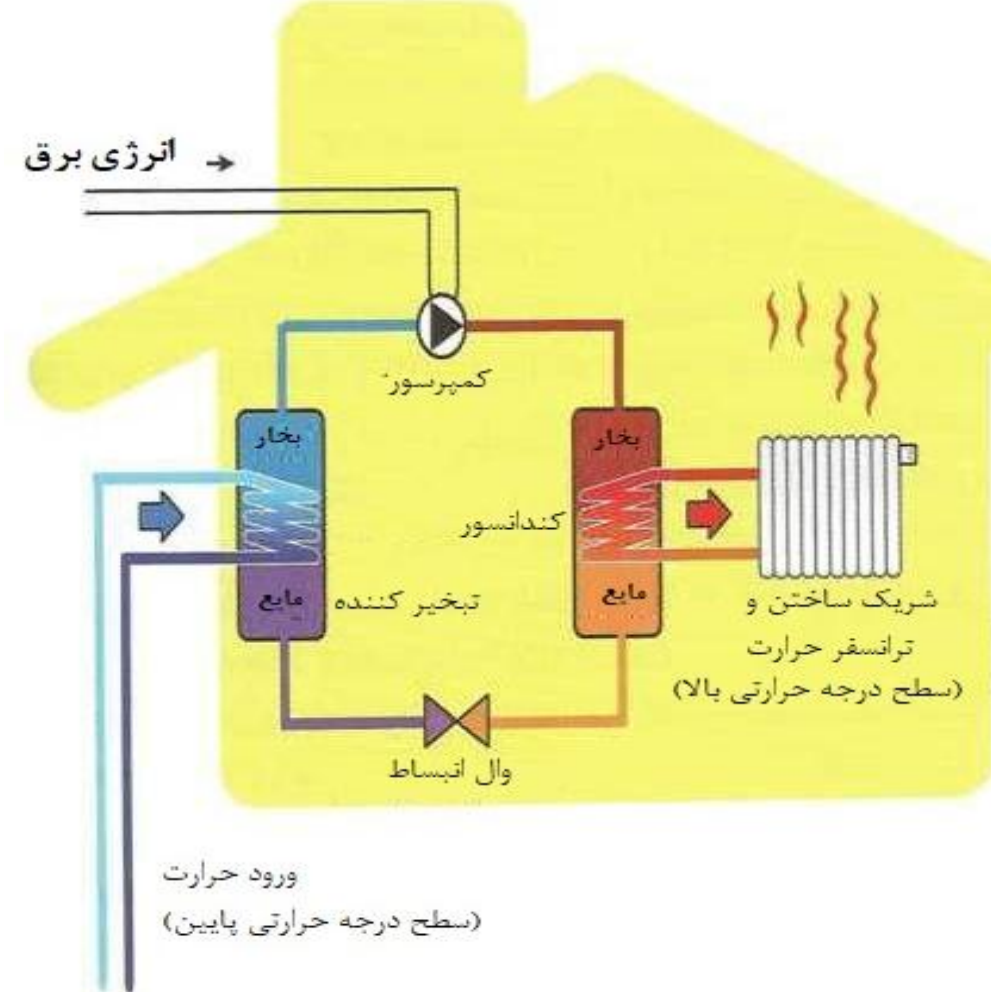

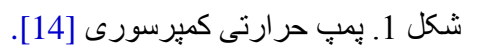

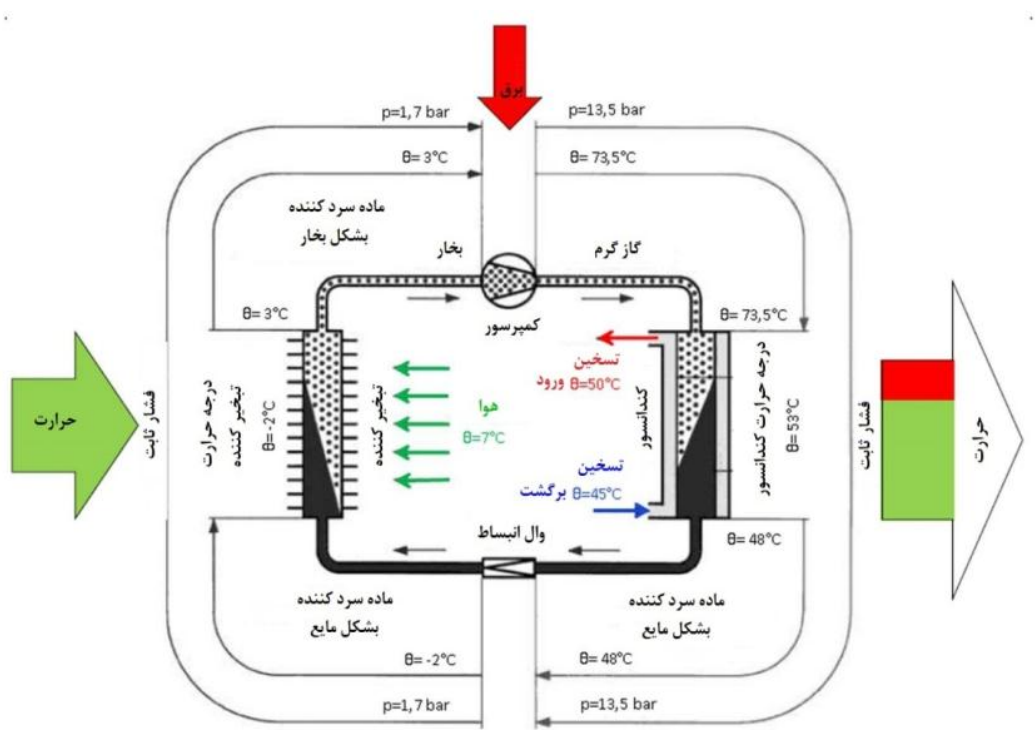


شكل 2. يمب حر ارتى با تعريف حالت هاى ماده سرد كننده. در هنحام تغيير حالت بهره بردارى يا استفاده از ماده سرد كننده ديكر ، نتايج متفاوت خو اهد بود

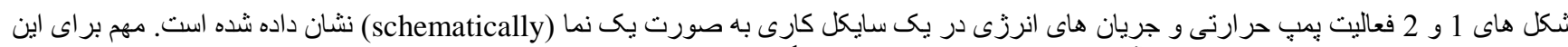

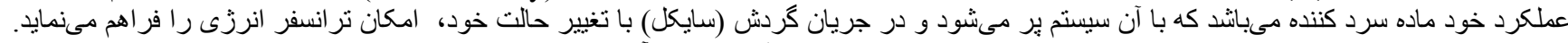

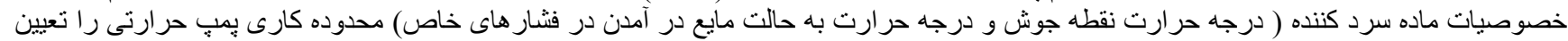

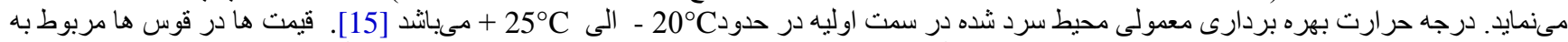

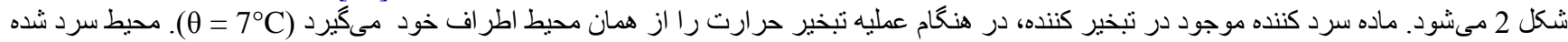

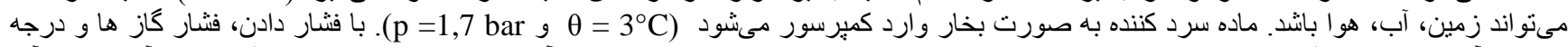

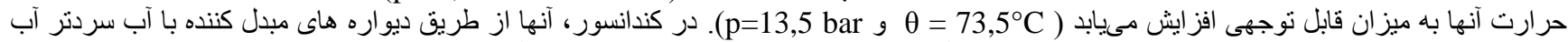

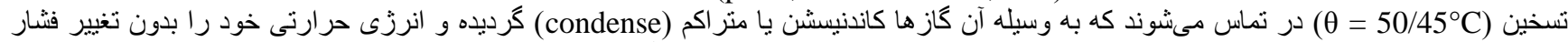

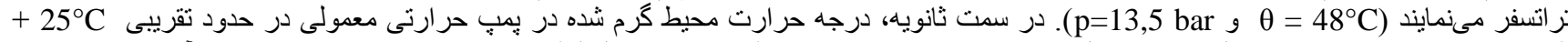

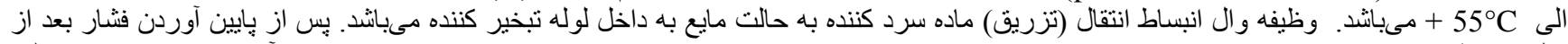

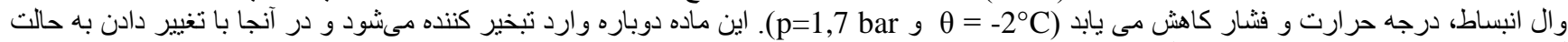

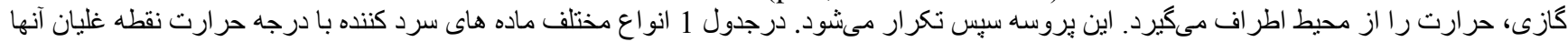

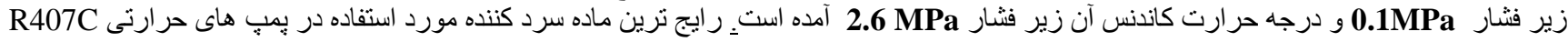

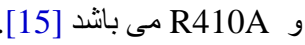

جدول 1. مقادير درجه حرارت ماده هاى سرد كننده متداول كه در يٌّب حر ارنى از آن استفاده مىشود [15].

\begin{tabular}{|c|c|c|c|}
\hline درجه حر ارت مايعات كاندنس تحت فشار 26 bar (2,6 MPa) & $\begin{array}{l}\text { نقطه غليان تحت فشار } 1 \text { bar (0,1 MPa) } \\
\end{array}$ & 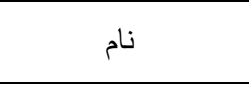 & علامت كذارى \\
\hline $86^{\circ} \mathrm{C}$ & $-30^{\circ} \mathrm{C}$ & ديكلروفلوئورومتان & R 12 \\
\hline $80^{\circ} \mathrm{C}$ & $-26^{\circ} \mathrm{C}$ & 1,1,1,2 طنرفلورثن & $\mathrm{R} 143 \mathrm{a}$ \\
\hline $70^{\circ} \mathrm{C}$ & $-42^{\circ} \mathrm{C}$ & بـ بِرويان & R290 \\
\hline $55^{\circ} \mathrm{C}$ & $-47^{\circ} \mathrm{C}$ & مخلوط فلوروكربن & R4040A \\
\hline $58^{\circ} \mathrm{C}$ & $-45^{\circ} \mathrm{C}$ & مخلوط فلوروكربن & $\mathrm{R} 407 \mathrm{C}$ \\
\hline $43^{\circ} \mathrm{C}$ & $-51^{\circ} \mathrm{C}$ & مخلوط فلوروكربن & $\mathrm{R} 410 \mathrm{~A}$ \\
\hline $114^{\circ} \mathrm{C}$ & $-12{ }^{\circ} \mathrm{C}$ & بوتان & R600A \\
\hline $60^{\circ} \mathrm{C}$ & $-33^{\circ} \mathrm{C}$ & آمونياك & $\mathrm{R} 717$ \\
\hline$-57^{\circ} \mathrm{C}$ & $-57^{\circ} \mathrm{C}$ & R744 & $\mathrm{R} 744$ \\
\hline $61^{\circ} \mathrm{C}$ & $-48^{\circ} \mathrm{C}$ & ي بروين & $\mathrm{T} 1070$ \\
\hline
\end{tabular}

2.3

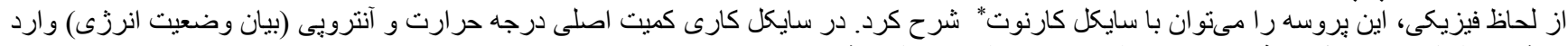

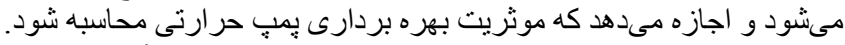

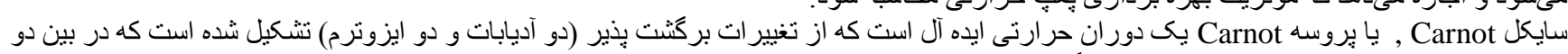
ظرف با درجه هاى حرارت مختلف صورت مىكيرد.

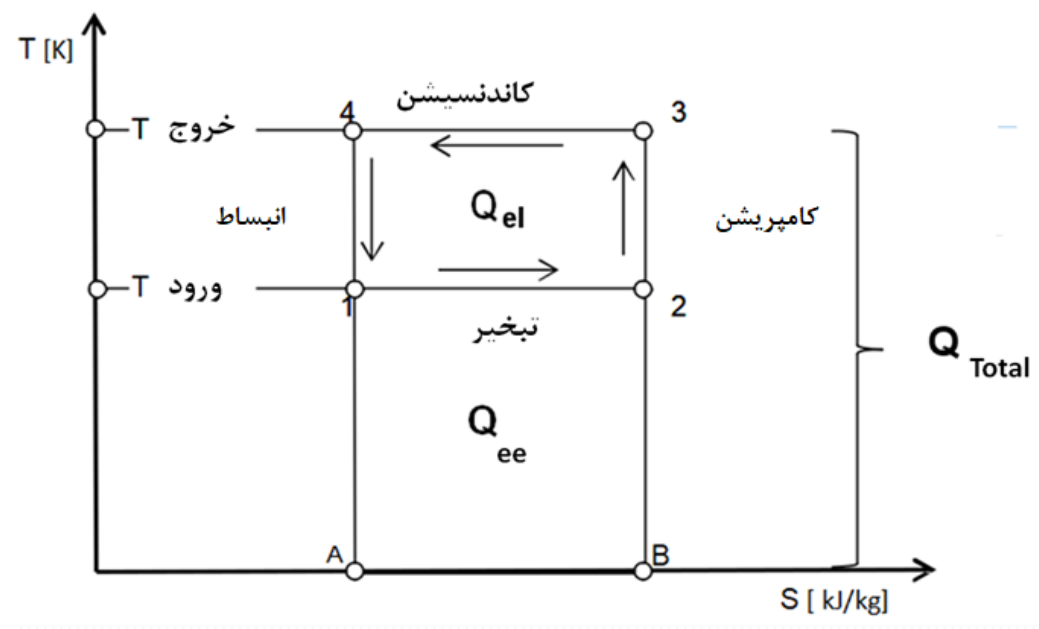

شكل 3. نمايش كر افيكى سايكل كارى نظرى بِّ حر ارتى [14, 16.

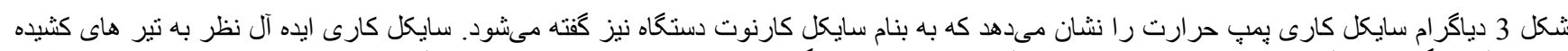

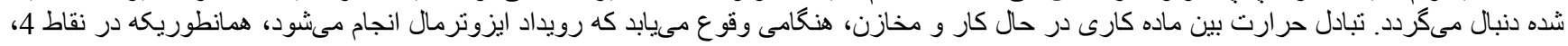




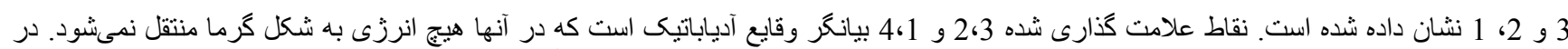

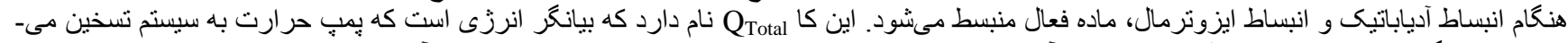

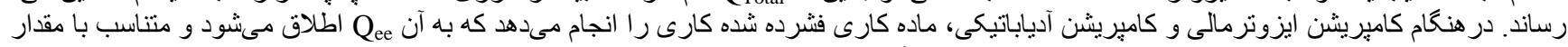

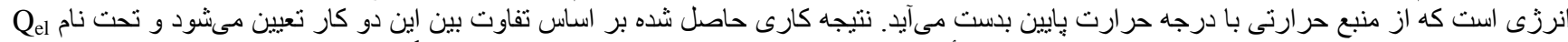

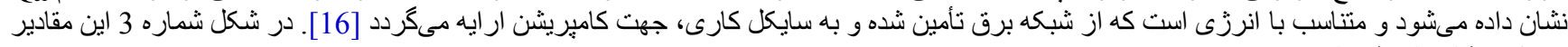
$(\mathrm{K})$

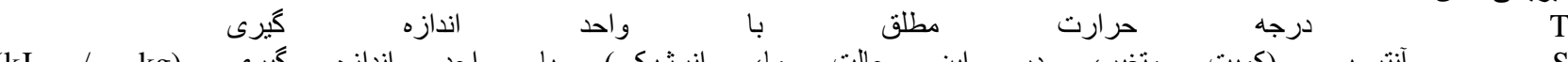

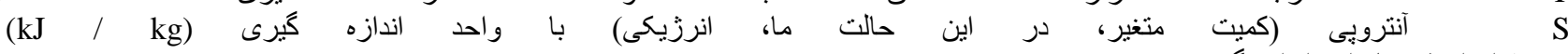

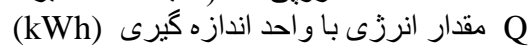

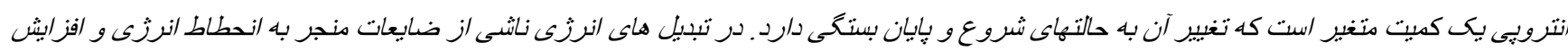
انتزروبيى مى شوند.

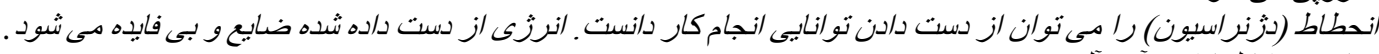

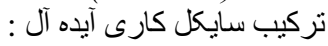
جهار فازسايكل كارى ايده آل بِّب حرارتى را نيز مىتوان به سادگى به شرح زير توضيع داد [17]:

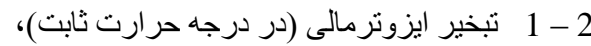

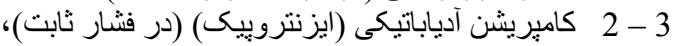

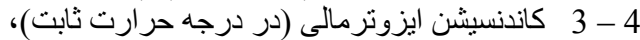
1 - 4 - انبساط آدياباتيكى (ايزنترويى) (در فثار ثابت).

$$
Q_{\text {Total }}=Q_{e e}+Q_{e l}
$$

مجموع انرزى عرضه شده از بٍِّ حرارتى به سبيتم

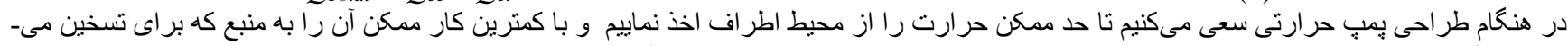

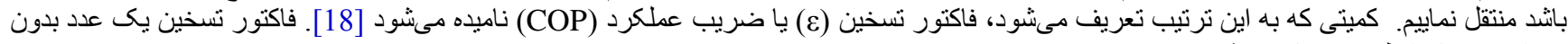

$$
\mathcal{E}=C O P=Q_{\text {Total }} / Q_{e l}=\left(Q_{e e}+Q_{e l}\right) / Q_{e l}=T_{\text {output }} /\left(T_{\text {output }}-T_{\text {input }}\right)
$$

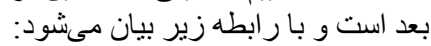

$$
\text { ut } \left.-T_{\text {input }}\right)
$$

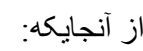

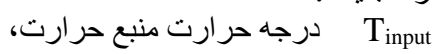

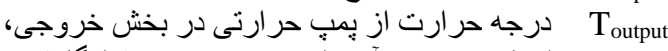

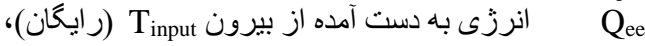

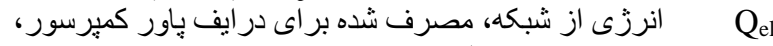

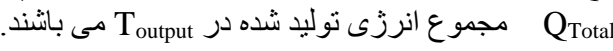

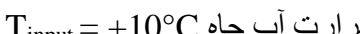

$\mathrm{T}_{\text {output }}=+50^{\circ} \mathrm{C}$ درجه حرارت سيستُ تسخين راد ادياتور $\varepsilon=\mathrm{T}_{\text {output }} /\left(\mathrm{T}_{\text {output }}-\mathrm{T}_{\text {input }}\right)=(273+50) /((273+50)-(273+10))=323 / 40=\underline{8,08}$ با ترانسفر حرارت، از نظر تئورى تو انستيم 8 برابر انرزى بيشترى را دريافت كنيم از آنجه در ورود از آن استفاده كرديم.

دثثال دوم

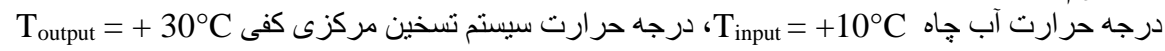
$\varepsilon=\mathrm{T}_{\text {output }} /\left(\mathrm{T}_{\text {output }}-\mathrm{T}_{\text {input }}\right)=(273+30) /((273+30)-(273+10))=303 / 20=\underline{15}$ / باترانسفر حرارت، از نظر تئورى تو انستيم 15 برابر انرزى بيشترى را دريافت كنيم از آنجه در ورود از آن استفاده كرديه.

محاسبات نشان مىدهد كه اختلاف درجه حرارت بين Tout

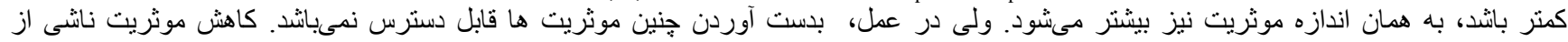

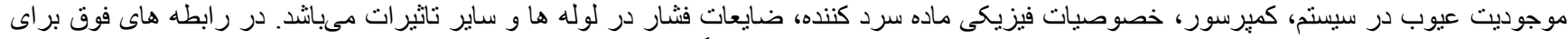

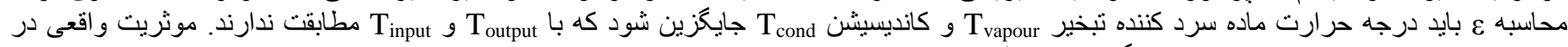
محدوده (3-5) = ع بوده و بايد با اندازه كيرى هائ طو لانى مدت تعيين شود.

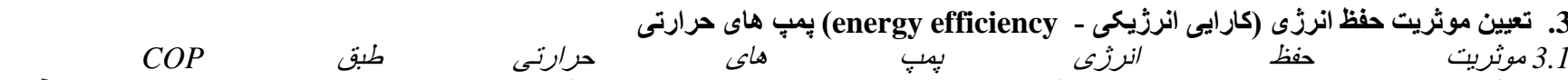

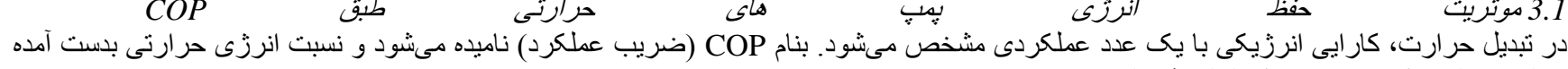

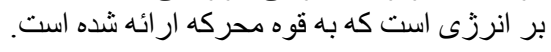

$$
\mathrm{COP}=\frac{\mathrm{Qt} 2}{\mathrm{Qe} 2}
$$

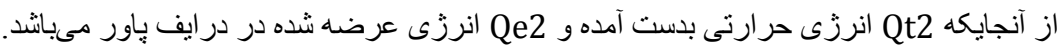

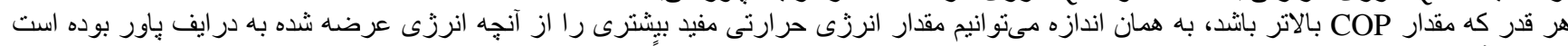

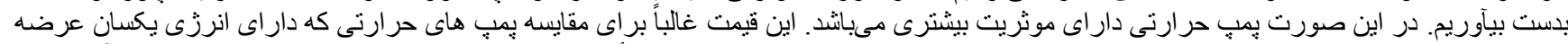

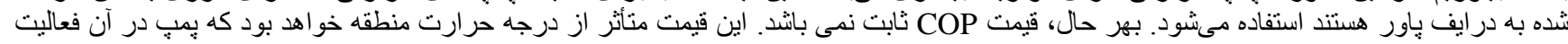




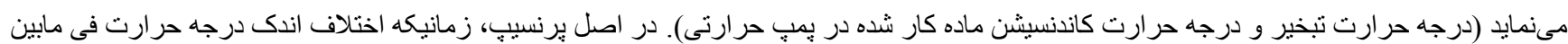

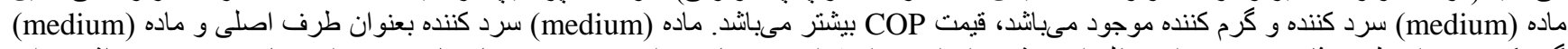

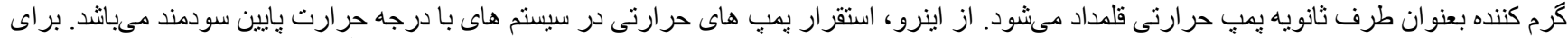

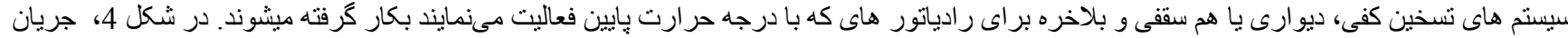

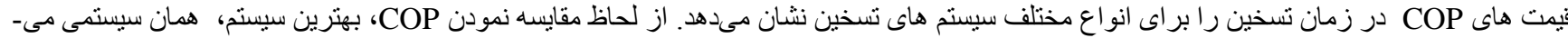
باثشد كه دار ایى بالاترين قيمت باثند.

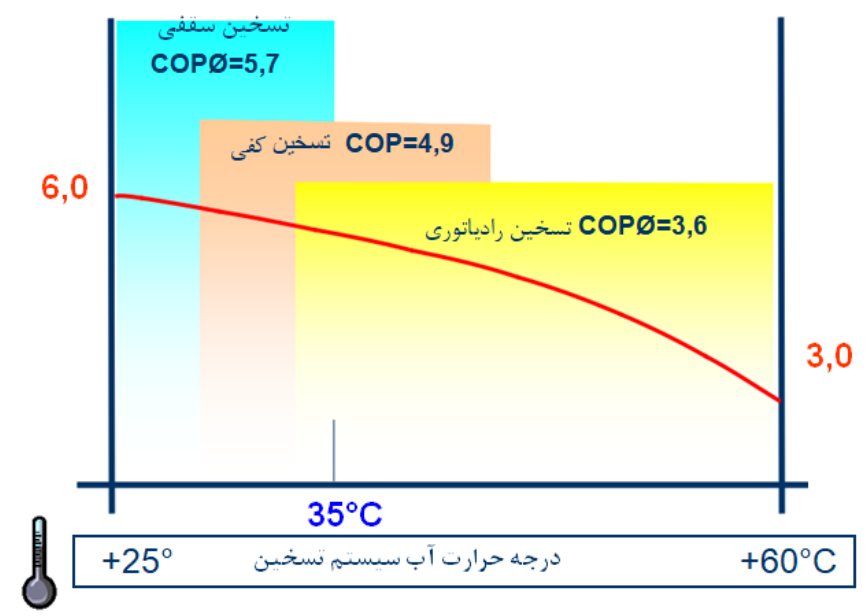

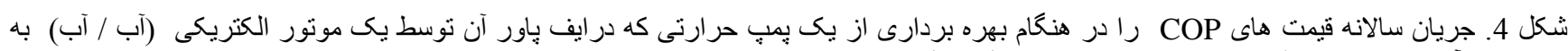

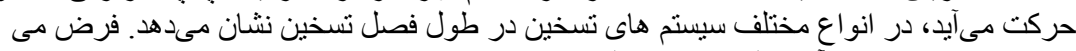

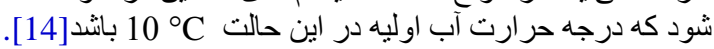

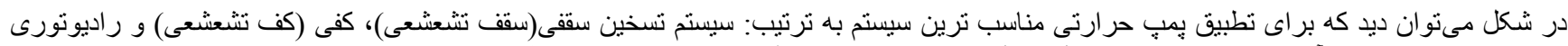

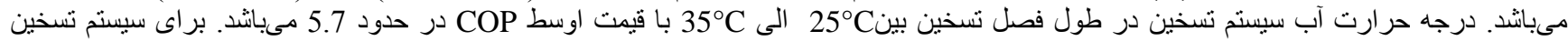

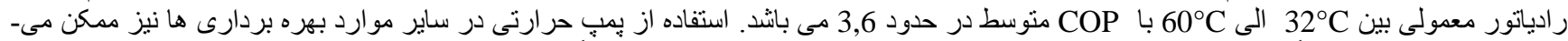

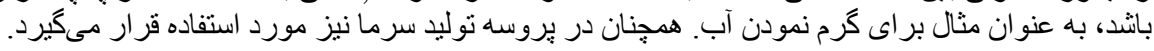

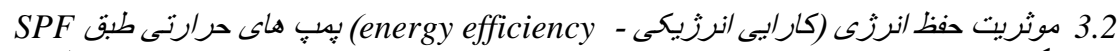

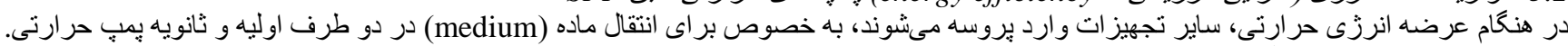

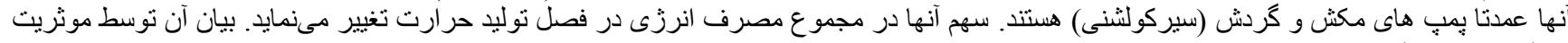

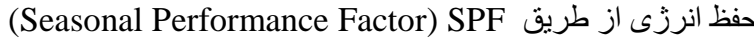

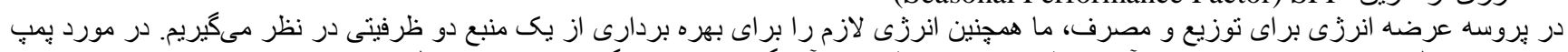

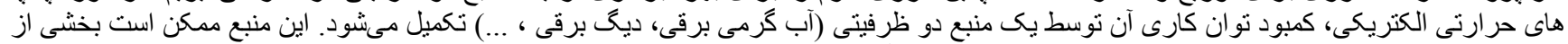

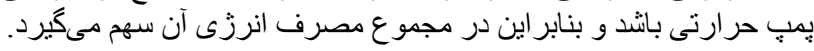

$$
\mathrm{SPF}=\frac{\mathrm{Qt} 2}{\mathrm{Qe} 1}
$$

در اينجا نسبت انرزى حر ارتى مفيد توليد شده (Qt2) بر انرزى عرضه شده در درايف ياور، منبع دو ظرفيتى و نوزيع انرزى (Qe1) را بيان مىنمايند.

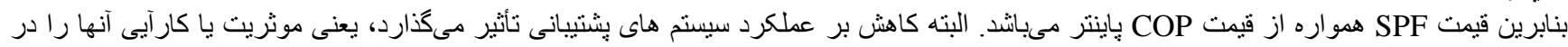

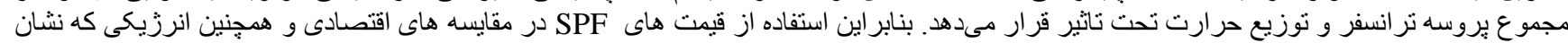

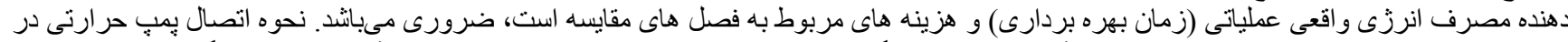

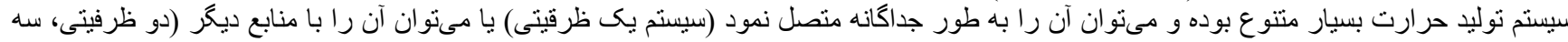




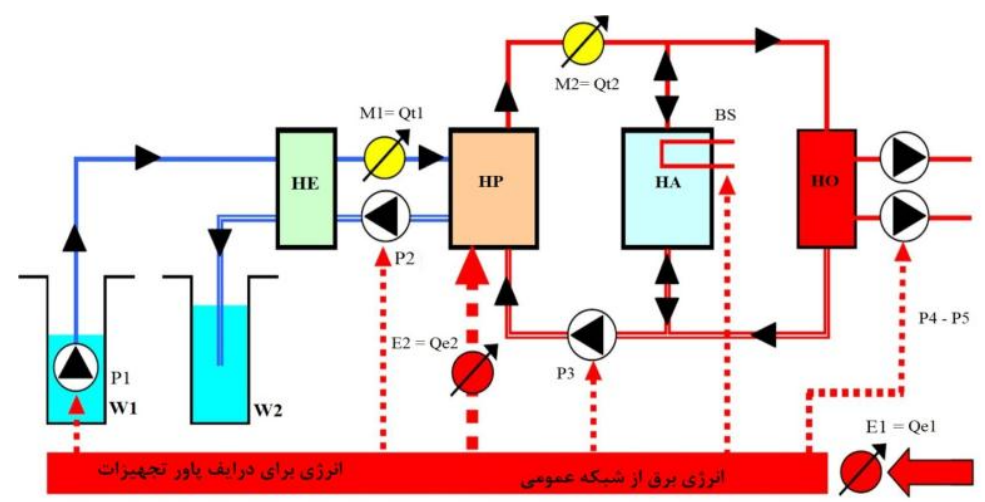

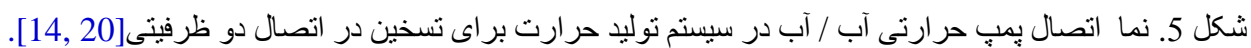

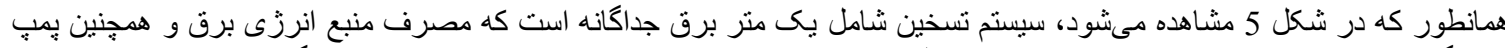

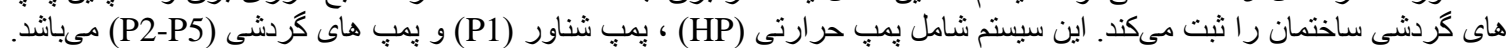

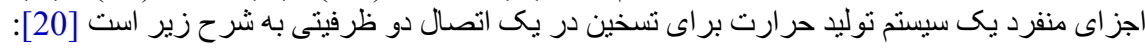

HE - Heat Exchanger

$\mathrm{HP}$ - Heat Pump - water/water , $0^{\circ} \mathrm{C} / 35^{\circ} \mathrm{C} \mathrm{COP} 3.8$; heat performance $95.3 \mathrm{~kW}$

HA - Heat Acumulation Tank

$\mathrm{HO}$ - Heated Object

M1 = Qt1 - Heat Meter

$\mathrm{M} 2=\mathrm{Qt} 2$ - Heat Meter

$\mathrm{E} 1=\mathrm{Qe} 1-$ Electricity Meter

$\mathrm{E} 2=\mathrm{Qe} 2$ - Electricity Meter

HA - Heating Storage Tank

BS - Bivalent/Backup heat source

W1-2 - Source/Suction well

P1 - Submersible Pump

P2-3 - Circulation Pumps - Engine Room

P4-5 - Circulation Pumps - Building

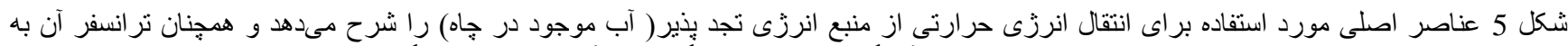

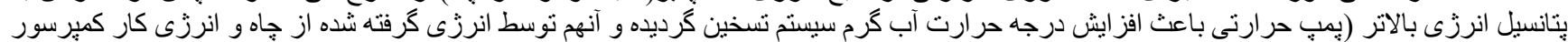

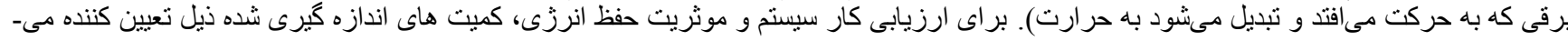

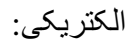

انرزى

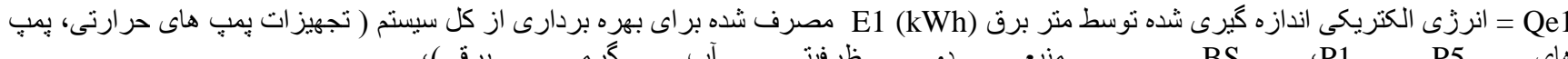
هاى

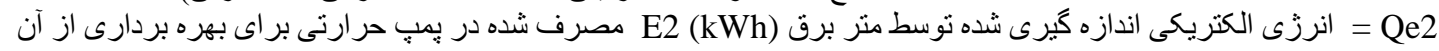

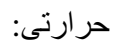

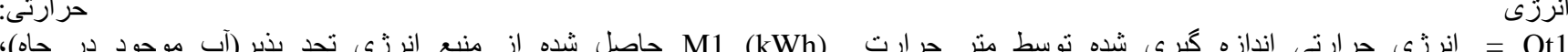

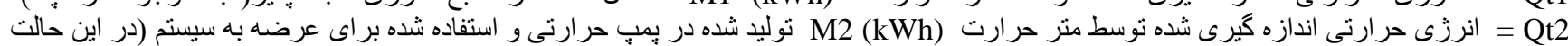

براى تسخين).

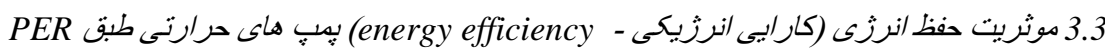

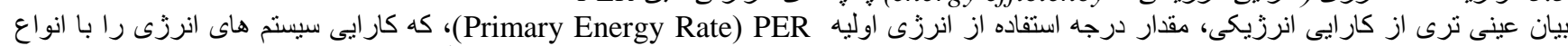

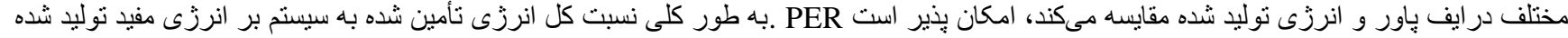

مئباشد [21].

$$
\mathrm{PER}=\frac{\mathrm{Qe} 1}{\mathrm{Qt} 2}
$$

در اينجا PER نسبت انرزى تأمين شده به سيستم (Qe1) و انرزى حر ارتى مفيد توليد شده (Qt2) را بيان مىنمايند.

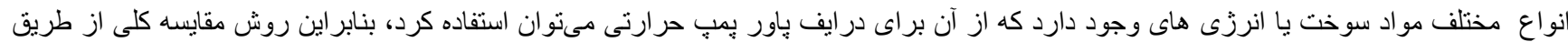

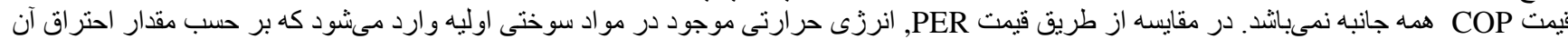

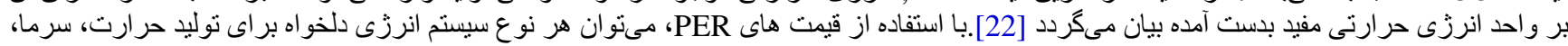

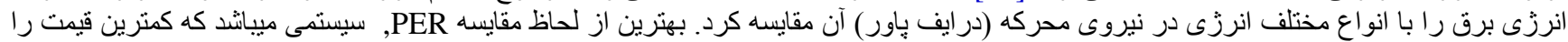




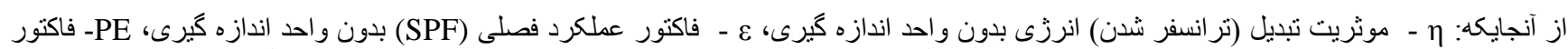

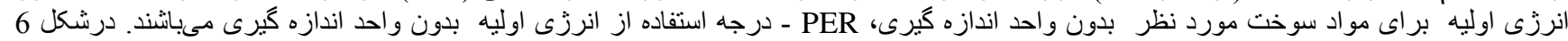

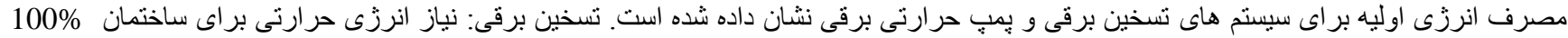

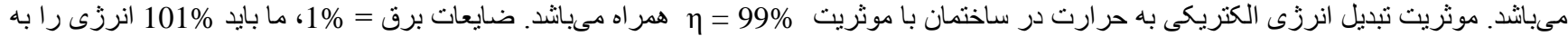

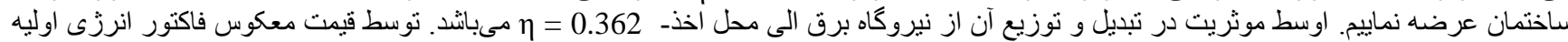

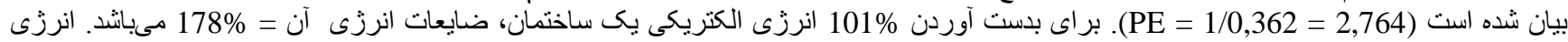

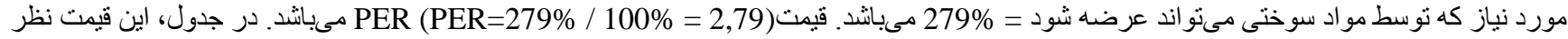

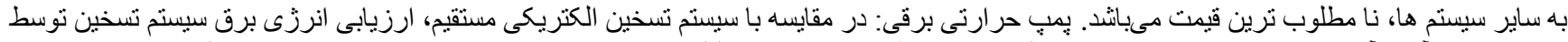

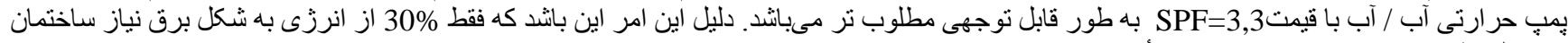
را از لحاظ مصرف انرزى براى تسخين تأمين مىنمايد.

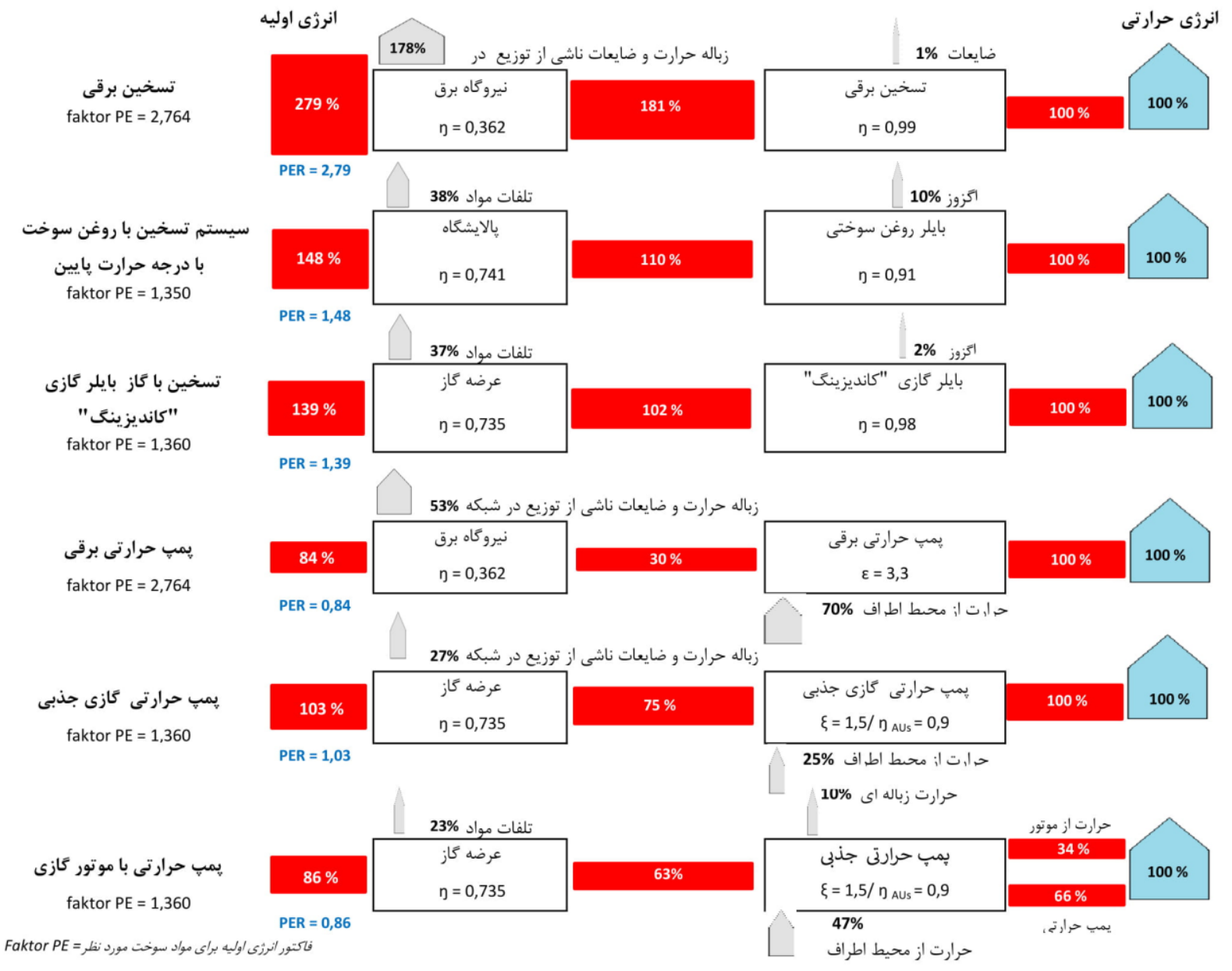

شكل 6. مصرف انرزى اوليه براى سيستم هاى مختلف تر اتنسفر حرارت[14].

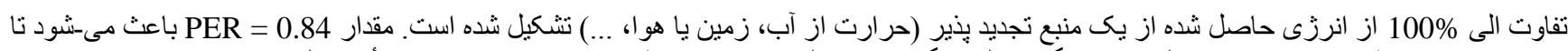

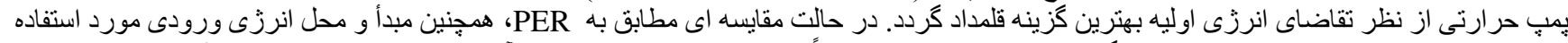

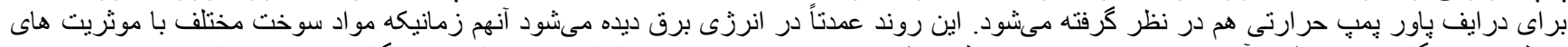

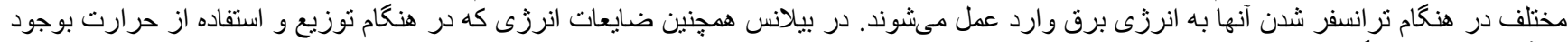

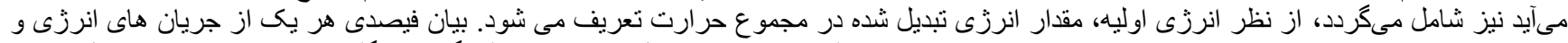

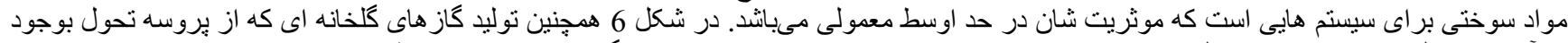

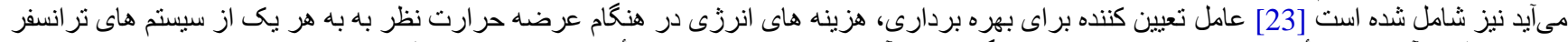

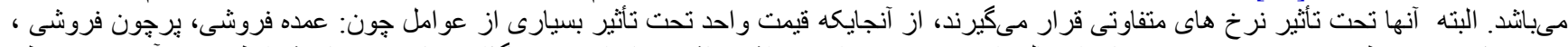

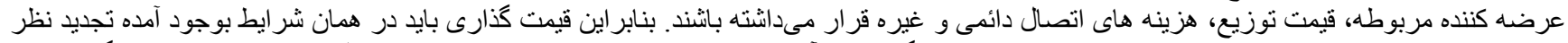

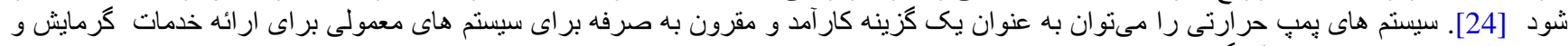

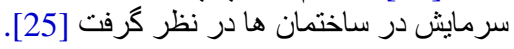

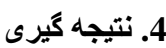

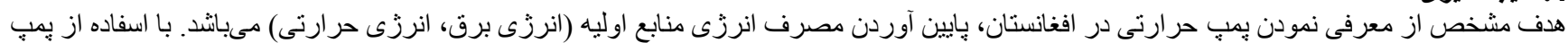

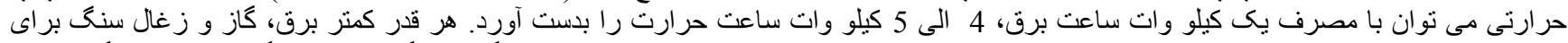

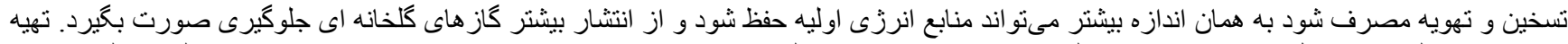

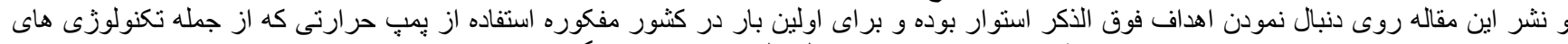

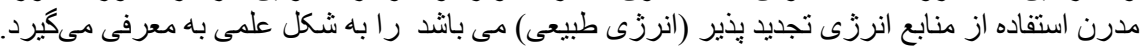




\section{References}

[1] K. J. Chua, S. K. Chou, and W. M. Yang, "Advances in heat pump systems," Applied Energy, vol. 87, pp. 3611-3624, 2010.

[2] F. Vranay, Z. Vranayova, and D. Ocipova, "Renewable energy source for today situation - geoexchange systems," vol. 266, pp. 99$107,2009$.

[3] J. Knaga and T. Szul, "Analysis of water-water type heat pump operation in a building object," Teka Komisji Motoryzacji $i$ Energetyki Rolnictwa, vol. 11, pp. 100-108, 2011.

[4] K. Tucki, M. Sikora, M. Karlikowska, and W. Bedkowski, "Selected issues of the technical analysis of a heat system," Using the Direct Evaporation Heat Pump, vol. 166, pp. 02-787, 2016.

[5] G. Floridesa and S. Kalogirou, "Ground heat exchanger - a review of systems, models and applications," Renewable Energy, vol. 32, pp. 2461-2478, 2007.

[6] A. Arsalis, S. K. Kær, and M. P. Nielsen, "Modeling and optimization of a heat-pump-assisted high temperature proton exchange membrane fuel cell micro-combined-heat-and-power system for residential applications," Applied Energy, vol. 147, pp. 569581, 2015. Available at: https://doi.org/10.1016/j.apenergy.2015.03.031.

[7] M. Štefanco, D. Košičanová, F. Vranay, M. Kušnír, and J. Lojkovics, "Principle of function of sorption heat pumps," Gasworks, Waterworks, Heating Engineer, Air Conditioning No.5-20142014.

[8] M. Kvakovský, L. Dedinská, and V. Čačková, "Utilization of heat pumps in building heating system," 2009.

[9] I. Staffell, D. Brett, N. Brandon, and A. Hawkes, "A review of domestic heat pumps," Energy \& Environmental Science, vol. 5, pp. 9291-9306, 2012.

[10] J. Pinka and B. Jagerova, "Possibilities of using heat pumps for heating family houses and flats," Acta Montanistica Slovaca, 2006.

[11] F. Vranay, Z. Vranayova, and M. Kovac, "Smart buildings versus ground energy and indoor technologies," vol. 106, pp. 235-240, 2009.

[12] Š. Antal, "Termodynamika, STU, Bratislava," 1992.

[13] J. Pinka, "Ecological and economic evaluation of the use of energy from hydrogeothermal sources and energy recovery by heat pumps," pp. 100-104, 2017.

[14] M. O. Temori, "Heat Pumps (2020), Mohammad Omar Teymouri, Heat Pumps (1399)," 2020.

[15] F. Vranay and M. Kusnir, "Renewable energy sources," Kosice, Slovakia, Faculty of Civil Engineering, Technical University of Kosice2017.

[16] D. Halliday, R. Resnick, and J. Walker, "Fyzika, Prometeus," 2006.

[17] A. Žeravík, "Stavíme tepelné čerpadlo," Euro Print, Spol. S R. O., 2003.

[18] M. Stefanco, D. Kosicanova, F. Vranay, M. Kusnir, J. Lojkovics, and C. Stone, "Heat pumps as a means of efficiency using renewable energy sources," IIn 14th International Multidisciplinary Scientific GeoConference SGEM 2014, vol. 1, pp. 267$274,2014$.

[19] M. Kušnír, F. Vranay, P. Kapalo, D. Košičanová, and Z. Vranayová, "The effective use of renewable energy sources in office building," Energy for Buildings, 9th ICEE, No. 267, 2014.

[20] M. Kušnír, D. Košičanová, Z. Vranayová, F. Vranay, and J. Lojkovics, "Zero energy balance proposal for office buildings," Technical Journal, vol. 2014, pp. 259-264, 2014.

[21] M. Jobb, M. Janovcová, M. Malcho, and J. Jandačka, "Influence of outdoor temperature on operating parameters of gas heat pump in real conditions," UNIZA2010.

[22] Act No. 86/2019, "Act No. 86/2019 Coll. 555/2005 Coll. on Energy Performance of Buildings and on Amendments and Supplements," 2019.

[23] M. Štefanco, D. Košičanová, F. Vranay, M. Kušnír, and J. Lojkovics, "Principle of function of sorption heat pumps, Gasworks, waterworks, heating engineer, air conditioning No.5-2014," 2014.

[24] F. Vranay and Z. Vranayova, "Influence of heat source choice on building energy certification process and CO 2 emissions. In International Conference Current Issues of Civil and Environmental Engineering Lviv-Košice-Rzeszów," ed Cham: Springer, 2019, pp. 541-548.

[25] N. Aste, R. S. Adhikari, and M. Manfren, "Cost optimal analysis of heat pump technology adoption in residential reference buildings," Renewable Energy, vol. 60, pp. 615-624, 2013. Available at: https://doi.org/10.1016/j.renene.2013.06.013. 